A Scientific/Technical Report for the

Advanced Detector Research Program

\title{
Selective Filtration of Gadolinium Trichloride for Use in Neutron Detection in Large Water Cherenkov Detectors
}

Closeout Award DE-FG02-07ER41494

September 1, 2007 - August 31, 2009

\author{
Principal Investigator: \\ Mark R. Vagins \\ Research Physicist \\ University of California, Irvine \\ 4129 Reines Hall \\ Irvine, CA 92697 \\ mvagins@uci.edu \\ (949) 824-2894
}

Prepared for the Division of High Energy Physics of the Office of Science (SC), U.S. Department of Energy 


\section{Contents}

1 Physics of Neutron Capture 2

1.1 In Pure Water . . . . . . . . . . . . . . . . . . . . 2

1.2 In the Presence of a Solute . . . . . . . . . . . . . . 2

2 Why Gadolinium Trichloride? 3

2.1 Solubility . . . . . . . . . . . . . . . . . 3

2.2 High Efficiency for Producing Observable $\gamma$-rays . . . . . . . . 4

2.3 Reasonable Cost . . . . . . . . . . . . . . . 5

2.4 Low Toxicity . . . . . . . . . . . . . . . . . 5

2.5 Few Unresolved Issues . . . . . . . . . . . . . 5 5

3 Physics Reach of WC Detectors with $\mathrm{GdCl}_{3} \quad 6$

3.1 Relic Supernova Neutrinos . . . . . . . . . . . . . . 6

3.2 Galactic Supernova Neutrinos . . . . . . . . . . . . . 7

3.3 Solar $\bar{\nu}_{e} \ldots \ldots \ldots$. . . . . . . . . . . . . . 8

3.4 Reactor Anti-neutrinos . . . . . . . . . . . . . . . . 9

3.5 Atmospheric Neutrinos . . . . . . . . . . . . . . . . . . . . 10

3.6 Long Baseline Neutrinos . . . . . . . . . . . . . . . . . . 11

3.7 Proton Decay . . . . . . . . . . . . . . . . 11

4 Program of Study $\quad 12$

4.1 Water Transparency . . . . . . . . . . . . . . . . . 14

4.2 Water Filtration and $\mathrm{GdCl}_{3}$ Recirculation . . . . . . . . . 16

$4.3 \mathrm{GdCl}_{3}$ Removal .................. 20 


\begin{abstract}
Water Cherenkov detectors have been used for many years as inexpensive, effective detectors for neutrino interactions and nucleon decay searches. While many important measurements have been made with these detectors a major drawback has been their inability to detect the absorption of thermal neutrons.

We believe an inexpensive, effective technique could be developed to overcome this situation via the addition to water of a solute with a large neutron cross section and energetic gamma daughters which would make neutrons detectable. Gadolinium seems an excellent candidate especially since in recent years it has become very inexpensive, now less than $\$ 8$ per kilogram in the form of commercially-available gadolinium trichloride, $\mathrm{GdCl}_{3}$. This non-toxic, non-reactive substance is highly soluble in water. Neutron capture on gadolinium yields a gamma cascade which would be easily seen in detectors like Super-Kamiokande.

We have been investigating the use of $\mathrm{GdCl}_{3}$ as a possible upgrade for the Super-Kamiokande detector with a view toward improving its performance as a detector for atmospheric neutrinos, supernova neutrinos, wrong-sign solar neutrinos, reactor neutrinos, proton decay, and also as a target for the coming T2K long-baseline neutrino experiment. This focused study of selective water filtration and $\mathrm{GdCl}_{3}$ extraction techniques, conducted at UC Irvine, followed up on highly promising benchtop-scale and kiloton-scale work previously carried out with the assistance of 2003 and 2005 Advanced Detector Research Program grants.
\end{abstract}



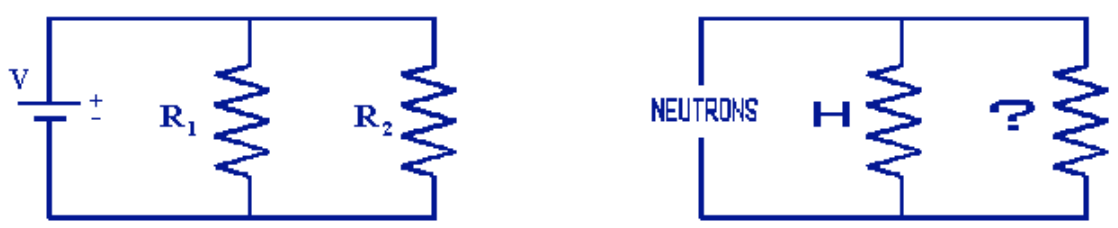

Figure 1: Resistors in parallel compete for electric current (left) just as multiple neutron-capturing elements in solution compete for neutrons (right).

\section{Physics of Neutron Capture}

\subsection{In Pure Water}

Neutrons liberated in water by the inverse beta reaction $\bar{\nu}_{e}+p \rightarrow e^{+}+n$ (and other processes) are quickly thermalized. On average it takes about twenty collisions with the water's free protons over the course of $\sim 10 \mu$ s to bring a neutron emitted with a few $\mathrm{MeV}$ down to room temperature $(0.025 \mathrm{eV})$.

Once thermalized, and after bouncing around for another $100 \mu \mathrm{s}$ or so, the neutron is captured by a proton or oxygen nucleus in the water. The cross sections for these capture reactions are 0.33 barns and 0.19 millibarns, respectively, so to first approximation every thermal neutron is captured on a free proton via the reaction $n+p \rightarrow d+\gamma$.

The resulting gamma has an energy of $2.22 \mathrm{MeV}$ and makes very little detectable light since the Compton scattered electron is close to Cherenkov threshold. Hence, in traditional water Cherenkov detectors (which tend to have trigger thresholds around $5 \mathrm{MeV}$ ) these neutron captures are generally not recorded and consequently there is no way to distinguish between neutrino interactions and anti-neutrino interactions on free protons.

\subsection{In the Presence of a Solute}

However, the situation can be quite different if the right substance is added to the water. As depicted in Figure 1, other elements will compete for the available neutrons in a manner mathematically identical to parallel resistors competing for electric current.

The relevant quantity for our case is the multiple of an element's neutron capture cross section with its concentration in the water; this product is 
analogous to $\frac{1}{R}$. For example, chlorine has a neutron cross section of 33 barns, exactly 100 times that of hydrogen. Since there are two hydrogen atoms per water molecule, we would have to introduce one chlorine atom for every fifty water molecules in order to balance their capture rates (this is equivalent to setting $R_{1}=R_{2}$ in Figure 1 ).

On the plus side, chlorine gives off a very energetic gamma cascade with a total energy of $8.6 \mathrm{MeV}$ when it captures a neutron - easily enough to be seen in just about any water Cherenkov detector. However, if we used regular table salt, $\mathrm{NaCl}$, as a soluble source of our chlorine, then we would have to add three kilotons of salt to Super-Kamiokande's fifty kilotons of water in order to observe $50 \%$ of the neutron captures (the $50 \%$ which continue to capture on hydrogen are still invisible, of course). This is because the molecular weight of $\mathrm{NaCl}$ is more than three times that of $\mathrm{H}_{2} \mathrm{O}$, so one molecule of $\mathrm{NaCl}$ to every fifty water molecules means that about $6 \%$ of salt by weight is required. Such a quantity is just at the limit of practicality. Even worse, if we wanted $90 \%$ capture efficiency on chlorine instead of $50 \%$ we would need to put about 27 kilotons of salt in Super-K... clearly impossible.

Therefore, we have turned to the best neutron absorber known - gadolinium [1]. Naturally occurring gadolinium (element number 64) has an absorption cross section of 49,700 barns, and has long been used in scintillator-based neutrino experiments near nuclear reactors $[2,3,4,5,6]$. As far as we can determine, however, it has never before been proposed for use in a water Cherenkov detector. The reason for this, as well as the reasons why we believe it's now time to utilize it, will be discussed in the next two sections.

\section{Why Gadolinium Trichloride?}

\section{$2.1 \quad$ Solubility}

Metallic gadolinium is essentially insoluble in water and so is unacceptable in its pure form for use in a large water Cherenkov detector. However, the chloride compound of this rare earth, gadolinium trichloride or $\mathrm{GdCl}_{3}$ (sometimes known simply as gadolinium chloride), is readily soluble in water. Dissolved concentrations of $\mathrm{GdCl}_{3}$ above $50 \%$ by mass in water are easily attainable, fully double $\mathrm{NaCl}$ 's maximum solubility limit of $25.3 \%$. 


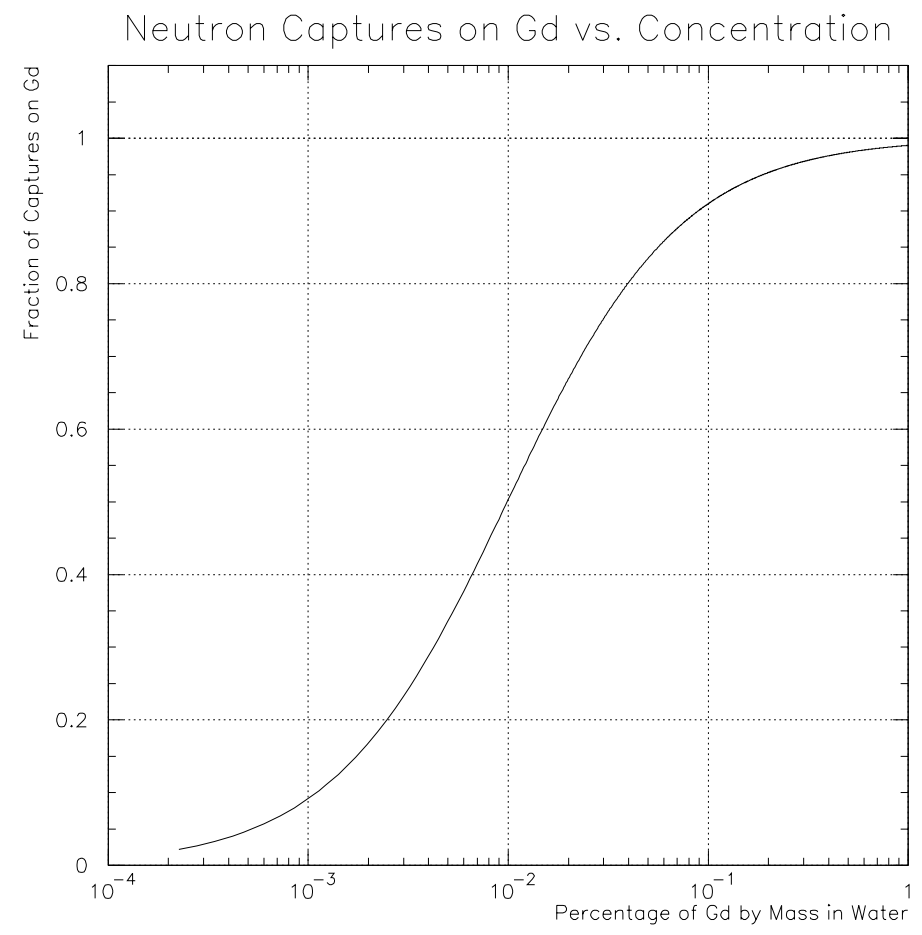

Figure 2: Fraction of neutrons visibly captured on gadolinium (instead of invisibly captured on hydrogen) versus the amount of $\mathrm{Gd}$ dissolved in light water.

\subsection{High Efficiency for Producing Observable $\gamma$-rays}

Gadolinium emits a healthy $8 \mathrm{MeV}$ gamma cascade when it captures a neutron. Like chlorine, this is enough energy to easily be seen in just about any large water Cherenkov detector. Unlike chlorine or $\mathrm{NaCl}$, however, the quantity of $\mathrm{GdCl}_{3}$ needed for high efficiency is not prohibitive. Working through the same calculation performed in Section 1.2 for $\mathrm{GdCl}_{3}$, we find that for $90 \%$ neutron capture efficiency on gadolinium we need to mix only 90 tons of $\mathrm{GdCl}_{3}$ into the 50 kilotons of Super-K's water: less than $0.2 \%$ by weight. Figure 2 tells the tale.

Another nice thing about $\mathrm{GdCl}_{3}$ is that nothing goes to waste: the chlorine will contribute to the neutron capture efficiency, if only an additional $0.2 \%$ or so (we now have three "resistors" in parallel). Still, it's good to know that we will not introduce any elements into the ultra-pure water which will 
be merely isotopic bystanders. Furthermore, higher efficiencies than $90.2 \%$ are likely achievable via higher concentrations of $\mathrm{GdCl}_{3}$.

\subsection{Reasonable Cost}

From a physics standpoint it certainly seems like $\mathrm{GdCl}_{3}$ is a nice compound to use for tagging neutrons, but can we afford 90 tons or more of it? As it turns out, there has been a dramatic revolution in the price of gadolinium over the past two decades. The opening of new mineral fields in the Gobi desert and the introduction of large-scale rare earth refining and purification technologies have caused the price to plummet three orders of magnitude in recent years. This could very well explain why no one had ever even proposed using gadolinium in a large detector before.

If we had tried to use gadolinium in Super-Kamiokande from day one the raw materials alone would have added $\$ 400$ million dollars to the cost of that $\$ 100$ million project. Today, acquiring 90 tons of $99.99 \%$ pure $\mathrm{GdCl}_{3}$ will cost us just under $\$ 400,000$.

\subsection{Low Toxicity}

Now that we can probably afford it, will it kill us? Many of the rare earths are moderately toxic, and there would be some difficulties involved in handling cubic-meter sized quantities of them.

Happily, $\mathrm{GdCl}_{3}$ is not only low in toxicity, but also, due in part to gadolinium's longstanding use as a contrasting agent for medical NMR scans and its recent discovery as a substance which blocks liver damage due to alcohol consumption, its physiological and toxicological effects are unusually well studied $[7,8,9,10]$ in both adult animal and human subjects. In the concentrations we are proposing to use in Super-Kamiokande, an adult human could in principle drink fourteen liters of this $0.2 \% \mathrm{GdCl}_{3}$ solution directly out of the tank every single day, indefinitely, with no discernible ill effects whatsoever. Nevertheless, all possible efforts will be made to avoid any release of $\mathrm{GdCl}_{3}$ into the environment.

\subsection{Few Unresolved Issues}

So, $\mathrm{GdCl}_{3}$ is a fairly well understood compound. But there are three main questions which still need to be addressed before anyone will approve mixing 
tons of it into any operating or future large water Cherenkov detector. Investigating these unresolved issues formed the essence of this proposal, and how we attempted to resolve them will be discussed in detail in Section 4 .

\section{Physics Reach of WC Detectors with $\mathrm{GdCl}_{3}$}

As attractive as $\mathrm{GdCl}_{3}$ looks from a neutron capture standpoint, there still must be some highly compelling physics reasons why we would want to modify a technology as mature and successful as that of large water Cherenkov detectors. These are the devices, after all, which in the last twenty years have discovered neutrino oscillations and neutrino mass [11], observed neutrinos from stellar collapse [12,13], pushed the limits on nucleon decay to theoretically unforeseen levels $[14,15]$, and provided confirmation of the Standard Solar Model [16, 17, 18].

There are indeed quite a few highly compelling physics reasons why we would now like to be able to see neutrons in water Cherenkov detectors. They are the topic of this section.

\subsection{Relic Supernova Neutrinos}

Some 170,000 years ago, a blue supergiant in the Large Magellanic Cloud

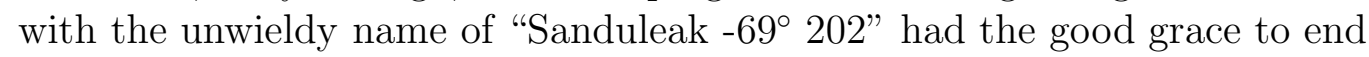
its life as a type II supernova. On February 23, 1987, the first radiation from the blast reached Earth. The blast, of course, became known as SN1987A, the first supernova observed anywhere near our galaxy since 1604 .

Three hours before the arrival of the light, a handful of neutrinos were seen via inverse beta decay, $\bar{\nu}_{e}+p \rightarrow e^{+}+n$ by one large water Cherenkov detector in Japan [12] and one in the US [13]. They saw only the Cherenkov light of nineteen emitted positrons: no information on the neutrons was collected.

While rather sparse, these first-of-their-kind observations absolutely energized the field of neutrino astrophysics. In fact, over the course of the following twenty years there has appeared, on average, one publication every ten days based on these nineteen events. In 2002, Masatoshi Koshiba of the University of Tokyo was awarded the Nobel Prize in Physics for his role as the head of the Kamiokande experiment which first saw the neutrino burst from SN1987A. 
Theorists and experimentalists alike wonder how we can get more data like this, as nearby supernovas are fairly rare events. However, all the neutrinos which have ever been emitted by every supernova since the onset of stellar formation suffuse the universe. These so-called "supernova relic neutrinos" [SRN], if observable, could provide a steady stream of information about not only stellar collapse and nucleosynthesis but also on the evolving size, speed, and nature of the universe itself.

Super-Kamiokande has recently conducted a search for these supernova relic neutrinos [19]. However, this study was strongly background limited, especially by the many low energy events below $19 \mathrm{MeV}$ which swamped any possible SRN signal in that most likely energy range. Consequently, this study could see no statistically significant excess of events and therefore was only able to set the world's most stringent upper limits on the SRN flux.

If it were possible to look for coincident signals, i.e., for a positron's Cherenkov light followed shortly and in the same spot by the gamma cascade of a captured neutron, then these troublesome backgrounds could be greatly reduced, and the energy range available for study greatly increased. SRN models vary, but in principle Super-K with $\mathrm{GdCl}_{3}$ should then see a few of these events every year. A much larger, future detector like the proposed Hyper-Kamiokande [20] would, with $\mathrm{GdCl}_{3}$ in its water, collect an amount of SRN events equal to what was seen twenty years ago from SN1987A every month or so.

\subsection{Galactic Supernova Neutrinos}

If we are fortunate enough to observe a nearby supernova in the coming decades, it would be most beneficial to have $\mathrm{GdCl}_{3}$ in the water of the large water Cherenkov detectors which are online when the resulting neutrino wave sweeps across the planet. This is because the most copious supernova neutrino signal by far $(\sim 80 \%)$ comes from inverse beta events. They are only produced by one of the six species of neutrinos and anti-neutrinos which are generated by a stellar collapse, and so if we could tag them individually by their follow-on neutron captures then we could extract the $\bar{\nu}_{e}$ time structure of the burst precisely. What's more, we could then subtract them away from the more subtle non- $\bar{\nu}_{e}$ signals, uncovering additional information that would otherwise be lost from this once-in-a-lifetime happening.

Being able to tag the $\bar{\nu}_{e}$ events would immediately double a water Cherenkov detector's pointing accuracy back to the progenitor star, since the elastic 
scatter events (about 3\% of the total) would no longer be sitting on a large background in angular phase space [21].

At the same time, this event-by-event subtraction would allow identification of the initial electron neutrino pulse from the neutronization of the infalling stellar matter, a key input in understanding supernova dynamics.

If the exploding star was big and quite close (we would also get an early warning of its impending collapse [22]. Approximately two days before exploding, the turn-on of silicon fusion in the core would raise the temperature of the star sufficiently that electron-positron annihilations within its volume would begin to produce $\bar{\nu}_{e}$ just above inverse beta threshold. In Super-K this would result in a sudden and persistent increase of our usual very low energy singles rate.

Finally, a gadolinium enriched Super-K would be sensitive to very late black hole formation following a supernova explosion anywhere within our galaxy, since the distinctive coincident inverse beta signals could be distinguished from the usual singles backgrounds. An abrupt cutoff of these coincident signals occurring even many minutes after the main burst would be the conclusive signature of a singularity being born.

\subsection{Solar $\bar{\nu}_{e}$}

Supposedly, the solar ${ }^{8} \mathrm{~B}$ neutrinos which have been measured by water Cherenkov detectors for years are all neutrinos. The Standard Solar Model [SSM] says that there should be no detectable solar anti-neutrinos produced at all [18]. However, no water Cherenkov detector to date has had the ability to directly differentiate between matter neutrinos and anti-matter neutrinos.

Adding gadolinium chloride to Super-Kamiokande or a similar detector would allow a very sensitive test of this fundamental SSM assertion. If there were as little as one solar $\bar{\nu}_{e}$ out of 10,000 solar $\nu_{e}$ 's, Super-K with $\mathrm{GdCl}_{3}$ would be able to spot it. Hyper-K with $\mathrm{GdCl}_{3}$ could do at least an order of magnitude better. Any observation of ${ }^{8} \mathrm{~B} \bar{\nu}_{e}$ 's from the Sun would have profound implications for our understanding of thermonuclear fusion and the processes by which stars generate their energy, and perhaps on our understanding of the boundary between matter and anti-matter itself. 


\subsection{Reactor Anti-neutrinos}

Nuclear power reactors produce prodigious quantities of low energy (below $8 \mathrm{MeV}$ ) anti-neutrinos. The neutrino was first discovered by using a reactor as a source [23], and in the years which followed the properties of $\bar{\nu}_{e}$ 's have been examined with increasing accuracy and precision by a series of scintillator-based short-baseline reactor experiments $[2,3,4,5,6]$. Finally, in what appeared to many to be the ultimate reactor neutrino experiment, the KamLAND experiment began operations a few years ago and has already provided strong evidence that the Large Mixing Angle MSW neutrino oscillation solution is the correct one to explain the long-standing Solar Neutrino Problem [24].

But KamLAND may not be the last word in reactor $\bar{\nu}_{e}$ 's, after all. If we were to introduce a $0.2 \%$ solution of $\mathrm{GdCl}_{3}$ into Super-Kamiokande, we could collect enough reactor anti-neutrino data to reproduce their first published results in just three days of operation. Their entire planned six-year datataking run could be reproduced by Super-K with $\mathrm{GdCl}_{3}$ in seven weeks, while Hyper-K with $\mathrm{GdCl}_{3}$ could collect six KamLAND-years of $\bar{\nu}_{e}$ data in just one day. Super-K would collect enough reactor $\bar{\nu}_{e}$ 's every day to enable it to monitor, in real time, the total reactor $\bar{\nu}_{e}$ flux. This means that, unlike KamLAND, it would not be dependent on the power companies which operate the reactors accurately reporting their day-to-day power output.

Although Super-K with $\mathrm{GdCl}_{3}$ will not be able to extract spectral information with an energy resolution as fine as that of scintillator detectors, it will have the unique ability to provide some $\bar{\nu}_{e}$ directional information via the emitted positrons' Cherenkov light [25]. This should, especially given the extremely high statistics involved, allow significantly tighter constraints to be placed on the solar neutrino oscillation parameters than any other method which could conceivably become operational before the close of the present decade, and possibly far beyond [26]. We would have these data in hand within months of the decision to introduce $\mathrm{GdCl}_{3}$ into Super-Kamiokande. Note that these plentiful reactor $\bar{\nu}_{e}$ events would not be confused with the comparatively rare relic supernova $\bar{\nu}_{e}$ 's or solar $\bar{\nu}_{e}$ 's because of the widely differing anti-neutrino energy ranges and spectra of the three processes. Figure 3 shows the expected spectrum of neutron-tagged positrons in a $\mathrm{GdCl}_{3}$ -enriched Super-K. The width of the band labeled "Supernovae" reflects the remaining allowed range of theoretical flux predictions for the as-yetunobserved relic supernova $\bar{\nu}_{e}$ 's. 


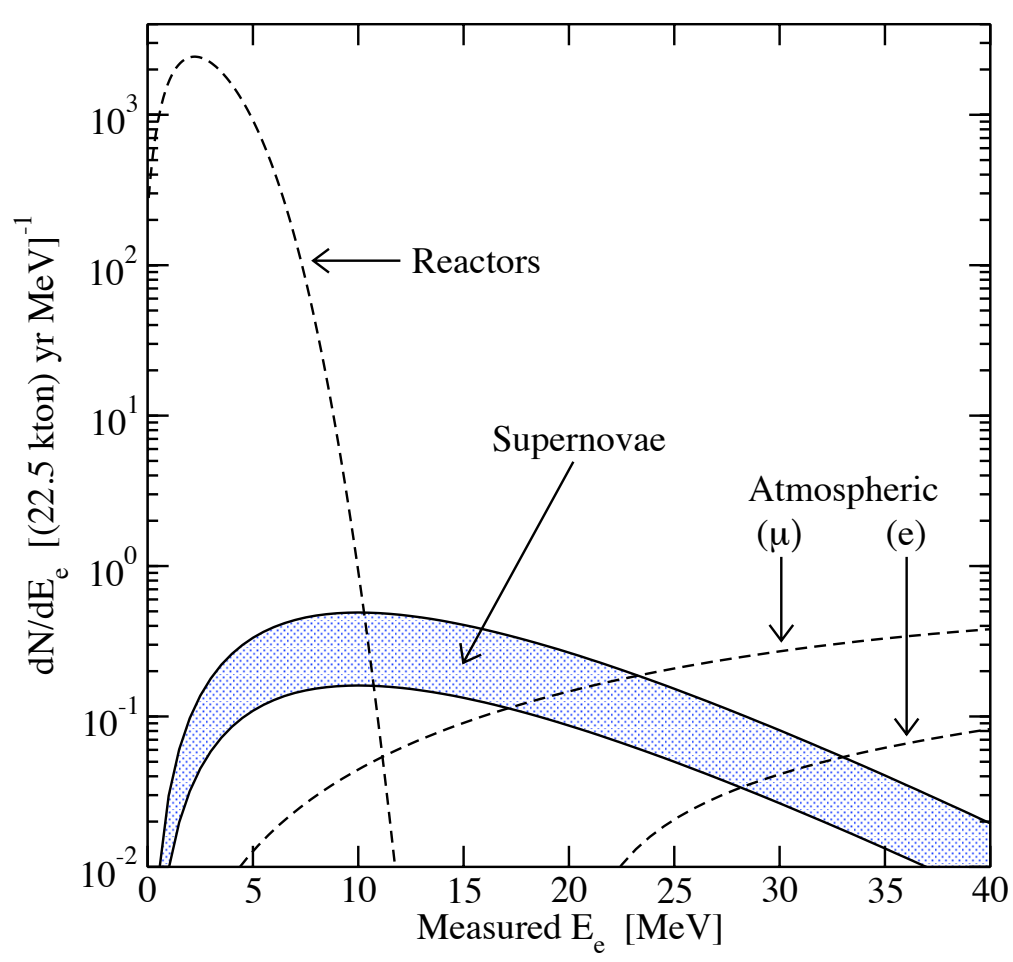

Figure 3: Expected positron spectrum tagged by neutron captures in a $\mathrm{GdCl}_{3}$ -enriched Super-Kamiokande. One year of data is shown, with SK's energy resolution and all known backgrounds taken into account. Note the huge reactor antineutrino signal, and the clear window for observing the relic supernova neutrinos between the reactor events and the tail end of the neutron-liberating atmospheric neutrino events.

\subsection{Atmospheric Neutrinos}

In addition to the observation of supernova neutrinos from SN1987A, large water Cherenkov detectors are probably best known for their Standard Modelshaking discovery of atmospheric neutrino oscillations [11]. One problem they suffer from, however, is a difficulty in differentiating between atmospheric neutrinos and atmospheric anti-neutrinos. This nagging flaw has led to some grandiose and likely unworkable schemes involving the placement of huge, powerful magnetic fields next to and/or within the next generation of experiments. As existing photomultiplier tubes will not operate in the presence of even the Earth's own weak field (Super-K's entire volume is carefully 
compensated via massive high-current coils which surround the detector and exactly cancel out the Earth's field) this method of matter/anti-matter identification appears quite impossible for water Cherenkov detectors.

With neutron-detecting $\mathrm{GdCl}_{3}$ in the water, however, the reactions $\bar{\nu}_{e}+$ $H \rightarrow e^{+}+n, \bar{\nu}_{\mu}+H \rightarrow \mu^{+}+n$, and $\bar{\nu}_{\tau}+H \rightarrow \tau^{+}+n$ are clearly distinct from the reactions $\nu_{e}+n \rightarrow e^{-}+p, \nu_{\mu}+n \rightarrow \mu^{-}+p$, and $\nu_{\tau}+n \rightarrow$ $\tau^{-}+p$, where the resulting proton generally produces no light. In an ideal world, by collecting a sample of neutron-tagged atmospheric anti-neutrino events we would be able to directly compare their flux and zenith angle dependence with that of the usual, mixed neutrino sample, thereby testing whether or not neutrinos and anti-neutrinos are behaving in the same way. In the real world, however, some neutrino interactions will eject neutrons from the oxygen nucleus, complicating matters. Still, with $\mathrm{GdCl}_{3}$ in the SK water it is likely that matter-enhanced and anti-matter enhanced samples could be collected and compared.

\subsection{Long Baseline Neutrinos}

Similar to the case of the atmospheric neutrinos, the ability to differentiate between neutrinos and anti-neutrinos would clearly benefit tests of oscillations and future measurements of the elusive neutrino mixing angle $\theta_{13}$ and what is perhaps the ultimate challenge of neutrino physics, the eventual measurement of the neutrino CP phase parameter [20].

Furthermore, elastic neutrino-neutron scattering, which will eject neutrons from oxygen nuclei in time with the beam spill, should provide a brandnew total flux normalization for the T2K beam as it arrives at Super-K, since this $\mathrm{NC}$ process is unaffected by the flavor of the incident neutrinos.

\subsection{Proton Decay}

Large water Cherenkov detectors got their start as proton decay experiments (in Kamiokande the last three letters stood for "nucleon decay experiment," while in Super-Kamiokande the same letters stand for "neutrino detection experiment") and still provide by far the most sensitive measurements of the proton and bound neutron lifetimes $[14,15]$. The next generation of detectors such as Hyper-Kamiokande are designed to push these limits even farther, hopefully observing a positive nucleon decay signal at last. 
In order to positively identify such a vanishingly rare process, however, these detectors will have to do everything possible to keep their backgrounds in check. These backgrounds are almost entirely due to atmospheric neutrino interactions which can occasionally mimic a true nucleon decay signal. As the atmospheric neutrinos cannot be shielded, one must live with them and their interactions. While more detailed study is needed, we believe that gadolinium's ability to spot free neutrons, neutrons which should almost never be produced by true nucleon decay events, will provide a useful new background reduction technique for these physics frontier projects.

\section{Program of Study}

We were awarded a 2003 Advanced Detector Research Program grant (DoE Grant Number: DE-FG02-03ER41266) for benchtop-scale study of $\mathrm{GdCl}_{3}$. The results were very promising, so much so that we were then awarded a new 2005 Advanced Detector Research Program grant (DoE Grant Number: DE-FG02-05ER41386) for large-scale $\mathrm{GdCl}_{3}$ testing at the one kiloton water Cherenkov tank $[\mathrm{KT}]$ from the K2K long-baseline neutrino oscillation experiment, a 2\% working scale model of Super-Kamiokande at KEK.

Unfortunately, seven years of exposure to ultra-pure water (with no gadolinium) during the entire $\mathrm{K} 2 \mathrm{~K}$ experimental run ultimately rendered the KT unsuitable for precision $\mathrm{GdCl}_{3}$ studies. Large areas (about $20 \%$ of the total inner surface area) had not been properly painted back in 1998, leaving the iron surface exposed and, by 2005, covered with a thick layer of rust. This was rapidly lifted into solution by the $\mathrm{GdCl}_{3}$, and caused complicating effects.

Nevertheless, we learned a number of important things from putting 200 $\mathrm{kg}$ of $\mathrm{GdCl}_{3}$ into the kiloton detector in 2005:

- $\mathrm{GdCl}_{3}$ is easy to dissolve in water.

- The $\mathrm{GdCl}_{3}$ itself (i.e., in the absence of old rust) does not drastically affect the light collection. An initial $\sim 1.5 \%$ drop in total collected light was seen after the $\mathrm{GdCl}_{3}$ was added, but what fraction of that was due to the $\mathrm{GdCl}_{3}$ and what was due to the lifted rust was not clear.

- Choice of detector materials is critical with $\mathrm{GdCl}_{3}$. 
- The 20-inch Super-K PMT's operate well in conductive water. During four months of exposure to the $\mathrm{GdCl}_{3}$ none of these PMT's (out of 680) failed.

- Our RO-based Gd filtration system works as designed at 3.6 tons/hr and can easily be scaled up to higher (Super-K level) flows.

Note that the $\mathrm{GdCl}_{3}$ itself did not cause the rust. This has been checked with tabletop tests involving clean and pre-rusted iron samples soaked in $\mathrm{GdCl}_{3}$ solutions. As Super-K is made of high-grade stainless steel, not (badly) painted iron, we expect no such problems there. An internal report by Mitsui Heavy Industries, the Japanese shipbuilding company who built the Super-K tank, also supports this conclusion, and $\mathrm{GdCl}_{3}$-induced corrosion is now expected to be insignificant in SK.

In the previous section we discussed the many powerful physics advantages to adding $\mathrm{GdCl}_{3}$ to large water Cherenkov detectors. However, as we mentioned at the end of Section 2, there are still three key unresolved issues regarding this use of gadolinium chloride. They are as follows:

1. What specific effect, if any, does dissolved $\mathrm{GdCl}_{3}$ have on light propagation in water?

2. How can we recirculate and clean the water without simultaneously reducing its $\mathrm{GdCl}_{3}$ concentration?

3. When we need to remove tons of $\mathrm{GdCl}_{3}$ at the end of the experiment, how can we do so quickly and inexpensively?

We were awarded a 2007 Advanced Detector Research Program grant (DoE Grant Number: DE-FG02-07ER41494) for the study of these three topics. The results to date have been very promising, so much so that we feel it is now clearly time to take this work to the next level - detailed, scalemodel proof-of-principle testing with an eye to enriching Super-K itself.

We will now describe our 2007-2009 R\&D program (there was a nocost, one year extention granted to the original 2007-2008 ADR award) for answering these vital questions. 


\subsection{Water Transparency}

The most straightforward of the three things we wanted to investigate is the optical effect of adding gadolinium chloride to the ultra-pure water found in Super-Kamiokande and other such detectors. This water has an attenuation length of close to 100 meters, necessary for efficiently collecting Cherenkov light which has traveled up to 47 meters inside the tank from its point of origin before reaching a PMT. It is already known that some solutes, like $\mathrm{NaCl}$ in moderate concentrations, do not significantly affect water transparency, but this effect had not yet been studied in detail with $\mathrm{GdCl}_{3}$. If its introduction were to cause the water to become more than a few percent less transparent then the entire $\mathrm{GdCl}_{3}$ idea would be in serious trouble, and we'd have to go looking for another solute if we still wanted the many physics advantages of visible neutron capture.

While a $0.2 \%$ solution of $\mathrm{GdCl}_{3}$ looks clear to the naked eye, we are concerned with Cherenkov light and therefore primarily with wavelengths between $300 \mathrm{~nm}$ and $500 \mathrm{~nm}$. Even so, in principle the study seems simple: use either a tunable laser or a device like the Varian Cary 50 UV-VIS Spectrophotometer to pass light of the appropriate frequencies through two sample containers, one with pure water and one with our $\mathrm{GdCl}_{3}$ solution in it. After measuring their relative attenuations with a phototube or photodiode we should know right away if $\mathrm{GdCl}_{3}$ adversely impacts the water transparency where it matters.

But there are two complicating factors: first, we need to measure light attenuation, not light absorption. Light which scatters out of its original direction is of no use in a water Cherenkov detector, even if it eventually bounces back into plumb, as its time of arrival will be fatally (in terms of accurate event reconstruction) delayed. Most commercially available devices are made to look for light absorption, and they are never concerned with detours the light might have taken in getting to the sensor. Second, most such devices have test cells no larger than $10 \mathrm{~cm}$ in any physical dimension, clearly not sufficient to measure changes in 100 meter water at the $\pm 5 \%$ level.

After quickly confirming with two borrowed UV-VIS spectrophotometers that our $\mathrm{GdCl}_{3}$ solution was not simply opaque in the near UV, in 2004 we made use of a UCI-funded, custom-built four-meter-long water attenuation measuring apparatus located in the Super-K mine. However, this is a tricky measurement; we used extremely clean water directly drawn from the Super$\mathrm{K}$ tank, but even transporting such highly-degassified and -purified water 
across the room to the attenuation measuring device can introduce large changes in attenuation length due to rapid absorption of gases from the air. This complicating effect was demonstrated by measuring samples with no $\mathrm{GdCl}_{3}$ added.

Also, we had no way to pretreat the $99.99 \%$ pure $\mathrm{GdCl}_{3}$ to remove residual dust and other contaminants present even in such high-quality product. Recirculation through the usual SK water system would have taken care of this, but we obviously did not want to introduce the $\mathrm{GdCl}_{3}$ into the main SK system just yet. Note that even a very tiny amount of dust can dramatically affect 100 meter-scale water transparency. At any rate, we were able to ascertain that the $\mathrm{GdCl}_{3}$ and all of its associated impurities had an attenuation length greater than 58 meters. There is no doubt that this is a hard lower limit, and will surely improve after dust and gas removal.

In order to characterize the attenuation length of ultrapure water in the presence of various solutes, we have used the funds provided by this grant to develop and assemble an extremely sensitive light attenuation measuring device which we call "IDEAL" (Irvine Device Evaluating Attenuation Length). A schematic of IDEAL is shown in Figure 4.

IDEAL extracts the absolute light attenuation length of water (with and without dissolved gadolinium) from an exponential fit to the light intensity of a pulsed laser beam as a function of the height of the column of water inside a 6.5 meter long vertical pipe. Six semiconductor laser pointers (whose wavelengths are $360 \mathrm{~nm}, 405 \mathrm{~nm}, 478 \mathrm{~nm}, 532 \mathrm{~nm}, 595 \mathrm{~nm}$, and $650 \mathrm{~nm}$ ) and a nitrogen gas laser $(337 \mathrm{~nm})$ are mounted at the top of the pipe. A reflective neutral density filter splits the beam and steers it into the pipe. Two integrating spheres [IS] read out by silicon photodiodes [PD] measure the intensity before and after the light is injected, thereby allowing fluctuations in input intensity to be properly normalized. Since the variation in received light intensity can quite small, especially in the case of high-quality water, much effort has gone into understanding and reducing systematic uncertainties.

IDEAL is now performing extremely well, and is capable of measuring ultrapure water with (wavelength-dependent) attenuation lengths far in excess of 100 meters. This is currently the most sensitive and accurate measuring device of its kind, allowing us to fine-tune our water filtration system described in the next section - for maximum effect.

The attenuation lengths at various wavelengths, both of ultrapure water and a $0.8 \%$ solution of $\mathrm{GdCl}_{3}$ ( $4 \mathrm{X}$ that required to realize the Gd-loading of SK), as measured by IDEAL are shown in Figure 5. We have demonstrated 
IDEAL: Irvine Device Evaluating Attenuation Length

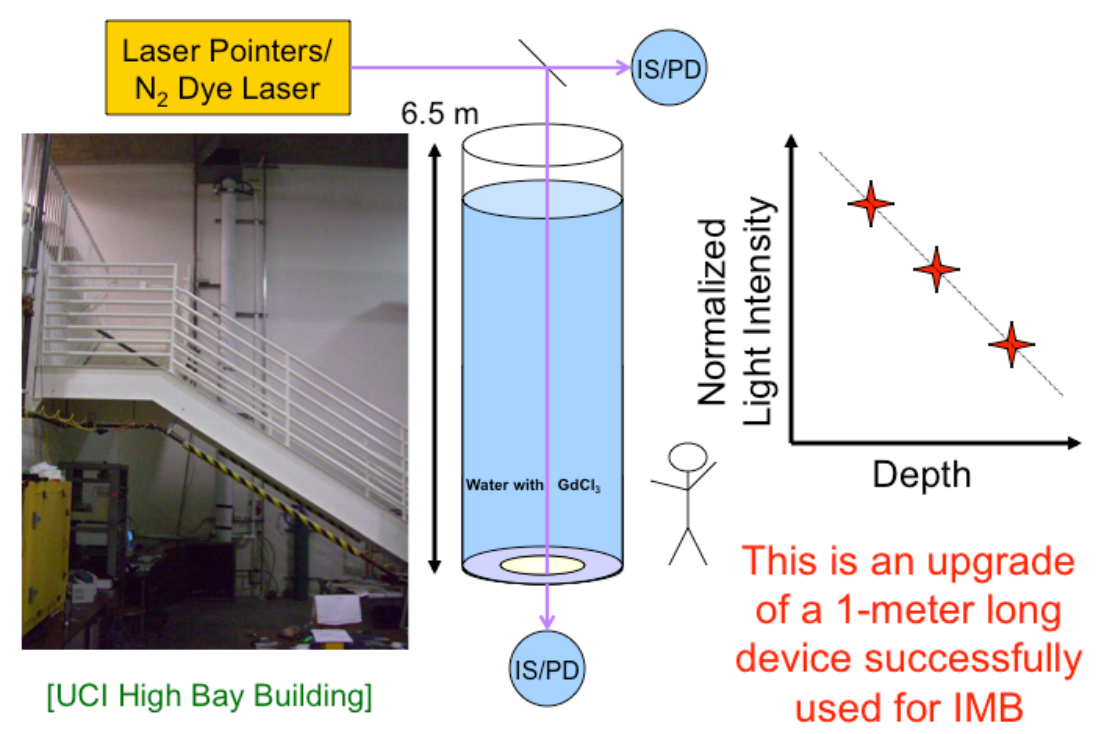

Figure 4: IDEAL, a much-improved version of a similar device which was first used by the Irvine Michigan Brookhaven [IMB] proton decay and neutrino experiment during the 1980's.

that attenuation of the $\mathrm{GdCl}_{3}$ will in fact be acceptable, a finding of vital importance on the road toward enriching SK with gadolinium.

\subsection{Water Filtration and $\mathrm{GdCl}_{3}$ Recirculation}

The second topic of investigation we have undertaken is discovering how to maintain acceptable water purity and constant $\mathrm{GdCl}_{3}$ concentrations while continuing to recirculate the water and to remove all other contaminants.

Small amounts of materials steadily leach into the water in a water Cherenkov detector, and in order to keep the water transparency close to 100 meters the water must be continuously recirculated through an elaborate water purification system. This system removes particulate matter, degassifies the water, kills and sweeps out biological agents, and eliminates ions. The concern in this case is that any $\mathrm{GdCl}_{3}$ introduced into Super-K or a similar detector will be rapidly extracted by the existing water system. Since the water in Super-K is "turned over" once per month, we would like to avoid 


\section{$\mathrm{GdCl}_{3}$ Solution}

\begin{tabular}{|l|l|l|}
\hline & pure $\mathrm{H}_{2} \mathrm{O}$ & $0.8 \% \mathrm{GdCl}_{3}$ \\
\hline $337 \mathrm{~nm}$ & $125.8 \pm 5.9 \mathrm{~m}$ & $27.74 \pm 0.26 \mathrm{~m}$ \\
\hline $360 \mathrm{~nm}$ & $210 \pm 21 \mathrm{~m}$ & $33.00 \pm 0.23 \mathrm{~m}$ \\
\hline $405 \mathrm{~nm}$ & $134.2 \pm 6.6 \mathrm{~m}$ & $66.8 \pm 0.9 \mathrm{~m}$ \\
\hline $478 \mathrm{~nm}$ & $65.1 \pm 5.6 \mathrm{~m}$ & $69.8 \pm 3.2 \mathrm{~m}$ \\
\hline $532 \mathrm{~nm}$ & $21.54 \pm 0.63 \mathrm{~m}$ & $21.08 \pm 0.51 \mathrm{~m}$ \\
\hline $595 \mathrm{~nm}$ & $6.402 \pm 0.015 \mathrm{~m}$ & $6.422 \pm 0.014 \mathrm{~m}$ \\
\hline $650 \mathrm{~nm}$ & $2.821 \pm 0.007 \mathrm{~m}$ & $2.864 \pm 0.004 \mathrm{~m}$ \\
\hline
\end{tabular}

Figure 5: Measured water transparencies at the various IDEAL wavelengths for pure water and a $0.8 \%$ solution of $\mathrm{GdCl}_{3}$, four times the level required to realize the Gd-loading of SK. The effect of adding gadolinium, while not insignificant at $405 \mathrm{~nm}$ and below, is acceptable.

having to replenish three tons of gadolinium chloride every day, at a cost of $\$ 13,000$. Even if this cost was deemed acceptable, the logistics of adding replacement $\mathrm{GdCl}_{3}$ every day and dealing with a few-percent fluctuation in our neutron capture efficiency would be quite serious obstacles to overcome. Hence, we need to find a way to have the water system either selectively ignore the $\mathrm{GdCl}_{3}$ entirely, or sweep it out and automatically re-introduce it once the water has been cleansed of all other substances.

In 2004 we built a small model of the Super-K water system at UCI. After experimenting with a variety of technologies and techniques, we found that by using a combination of reverse osmosis [RO] and electrodeionization [EDI] about $99.99 \%$ of the dissolved $\mathrm{GdCl}_{3}$ could be extracted and reintroduced to the main holding tank on each pass through the system. This would lead to a loss of no more than 300 grams per day out of 90 tons of $\mathrm{GdCl}_{3}$ in Super-K. RO by itself (an easier technology than EDI to operate) is over 99.9\% effective - this is what was used in the kiloton test. 


\section{Membrane-based Filtering Technologies}

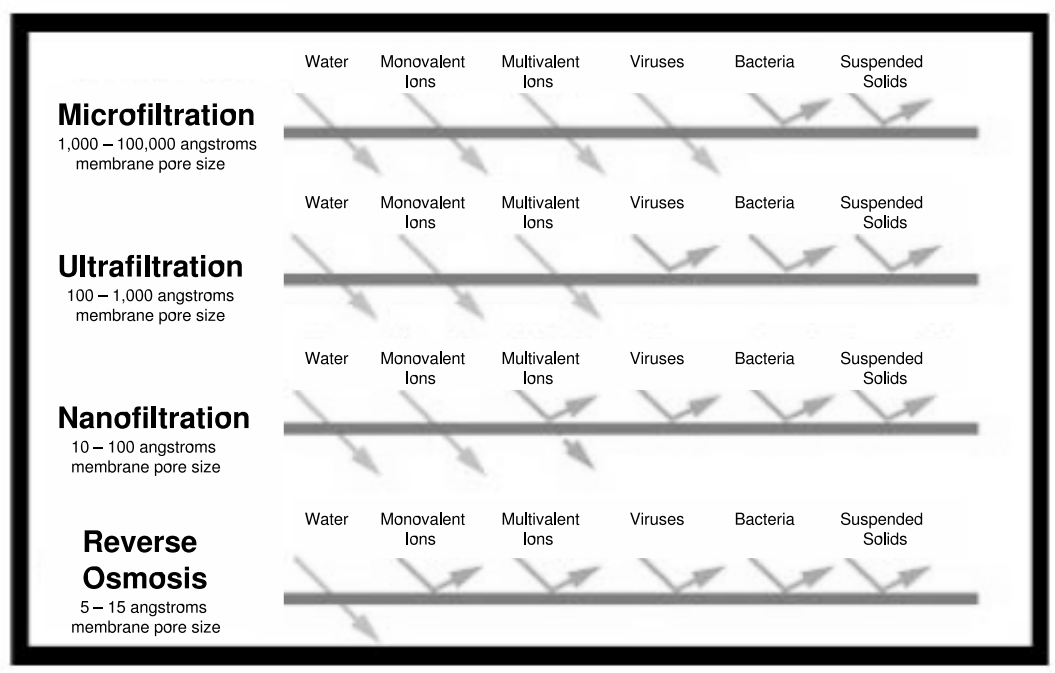

Figure 6: Comparison of several commercially available membrane-based filtering technologies. Note that Super-K already employs both ultrafiltration and reverse osmosis in its existing water system.

However, we are worried that other, unwanted substances may also be reintroduced into the tank by this scheme as well. To address this concern, the next step in our filtration studies - enabled by this grant -was the construction of something entirely new: a "band-pass filter" for $\mathrm{GdCl}_{3}$.

The essential idea is as follows: there are a variety of commercially produced, membrane-based filters on the market. Rated by the size of pores in the membrane, they reject contaminants larger than these holes, while passing those which are smaller into the product water stream. See Figure 6 for a depiction of the different filter membranes and their effective pore sizes.

By using a suitable sequence of filters, and by introducing nanofiltration $[\mathrm{NF}]$, a new membrane intermediate in pore size between reverse osmosis (which rejects all $\mathrm{GdCl}_{3}$ and everything larger) and ultrafiltration (which passes all $\mathrm{GdCl}_{3}$ and everything smaller), we hypothesized that a system could be assembled that selectively extracts $\mathrm{GdCl}_{3}$ from the water stream and returns it to the tank, while allowing all other impurities to be removed 


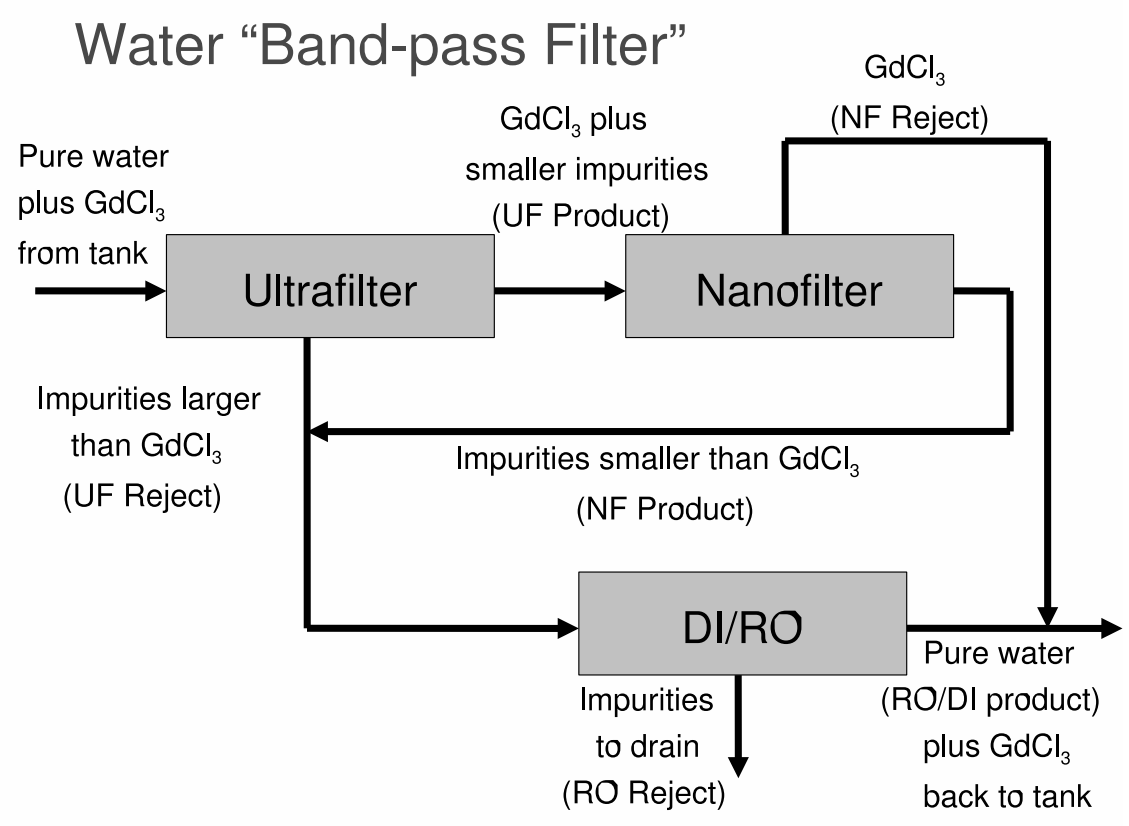

Figure 7: Schematic representation of a water "band-pass filter" for $\mathrm{GdCl}_{3}$. DI stands for deionization and RO stands for reverse osmosis.

via the usual combination of reverse osmosis and deionization. A prototype version of such a system, shown schematically in Figure 7, was developed and assembled at UCI and tested using the water transparency device (IDEAL) described above.

Once the proper membranes were put together in sequence this new approach proved to be very powerful. We found that a single stage of nanofiltration extracted $99.85 \%$ of the gadolinium from the circulating water and returned it to the test tank, while adding a second NF stage in series resulted in $>99.994 \%$ recovery of gadolinium. Equally important, the NF stage of the band-pass filter did indeed allow all undesirable, transparency-destroying impurities to be passed and subsequently removed by later stages.

The development of the selective band-pass filter represents a significant breakthrough in water filtration technology, and will undoubtedly affect the course, design, and results of all future water Cherenkov projects. 


\section{3 $\quad \mathrm{GdCl}_{3}$ Removal}

Any large water Cherenkov experiment which employs $\mathrm{GdCl}_{3}$ to enable neutron detection will eventually, either during planned maintenance or at the conclusion of the experiment, need to remove the $\mathrm{GdCl}_{3}$ from solution in as quick and cost-effective a manner as possible.

During our work with the kiloton detector we demonstrated that normal deionization resins are extremely effective in capturing $\mathrm{GdCl}_{3}$ from the water. Unfortunately, this is a very expensive way to remove large quantities of dissolved $\mathrm{GdCl}_{3}$, costing approximately ten times the price of the $\mathrm{GdCl}_{3}$ itself in terms of depleted resin. Even if $\$ 5,000,000$ worth of resin was deemed acceptable to remove 90 tons of $\mathrm{GdCl}_{3}$ from Super-K, handling several hundred tons of resin would be very cumbersome. Furthermore, such a removal approach will clearly not be acceptable for future, megaton-scale detectors. Therefore, we have also begun to investigate more efficient $\mathrm{GdCl}_{3}$ removal technologies.

We have determined that at a $\mathrm{pH}$ of 11 and above the dissolved gadolinium forms insoluble $\mathrm{Gd}(\mathrm{OH})_{3}$. A simple and inexpensive injection of sodium hydroxide, $\mathrm{NaOH}$, into the $\mathrm{GdCl}_{3}$ solution causes the near-instananeous dropout of $>99 \%$ of the gadolinium as this inert precipitate.

Our findings open up the possibility of employing any one of a variety of methods of removing this solid from the water, collecting it, and drying it for later disposal or recycling. These include the use of simple settling tanks, continuous-process cyclonic separation, filter presses, vacuum filtration, and perhaps even magnetic filtering.

Gadolinium is, rather surprisingly, ferromagnetic at the temperatures at which Super-K operates. While compounds of gadolinium will generally be less magnetic than the pure metallic form this unusual property might still be exploited to selectively sweep the precipitating $\mathrm{Gd}(\mathrm{OH})_{3}$ out of solution immediately after its formation.

\section{References}

[1] Antineutrino Spectroscopy with Large Water Cherenkov Detectors, J.F. Beacom and M.R. Vagins, Phys.Rev. Lett. 93, 171101 (2004)

[2] Search For Neutrino Oscillations In An Experiment In The Reactor Of The Rovno Nuclear Power Plant, A. I. Afonin et al., JETP Lett.42, 
285-288 (1985)

[3] Neutral Current Limit And Future Prospect At A Fission Reactor, Gurr, H. S., Reines, F., Sobel, H. W., Phys. Rev. Lett. 33, 179-181 (1974)

[4] Limits On Neutrino Oscillations From The Chooz Experiment, M. Apollonio et al., Phys. Lett. B466, 415-430 (1999)

[5] Search For Neutrino Oscillations At The Palo Verde Nuclear Reactors, F. Boehm et al., Phys. Rev. Lett. 84, 3764-3767 (2000),

[6] Development Of A Gd Loaded Liquid Scintillator For Electron Antineutrino Spectroscopy, Piepke, A. G., Moser, S. W., Novikov, V. M., Nucl. Instrum. Meth. A432, 392-398 (1999)

[7] Toxicological And Pharmacological Effects Of Gadolinium And Samarium Chlorides, Haley, T.J., K. Raymond, N. Komesu and H.C. Upham, Brit. J. Pharmacol. 17, 526-532 (1961)

[8] Effects Of Gadolinium Chloride On The Rat Lung Following Intratracheal Instillation, S. Yoneda, N. Emi, Y. Fujita, M. Omichi, S. Hirano and K.T. Suzuki, Fundam. Appl. Toxicol. 28, 65-70 (1995)

[9] Pulmonary Macrophage Function In Systemic Gadolinium Chloridepretreated Rats, Bannenberg G, Lundborg M, Johansson A., Toxicology Letters 80, 105-107 (1995)

[10] The Role Of Kupffer Cell Activation And Viral Gene Expression In Early Liver Toxicity After Infusion Of Recombinant Adenovirus Vectors, A. Lieber et al., J Virol 71, 8798-8807 (1997)

[11] Evidence For Oscillation Of Atmospheric Neutrinos, By the SuperKamiokande Collaboration (Y. Fukuda et al.), Phys. Rev. Lett. 81, 1562-1567 (1998)

[12] Observation Of A Neutrino Burst From The Supernova SN1987A, By the Kamiokande-II Collaboration (K. Hirata et al.), Phys. Rev. Lett. 58, 1490-1493 (1987)

[13] Observation Of A Neutrino Burst In Coincidence With Supernova SN1987A In The Large Magellanic Cloud, By the IMB Collaboration (R.M. Bionta et al.) Phys. Rev. Lett. 58, 1494-1497 (1987) 
[14] Search For Proton Decay Via $\mathrm{P} \rightarrow \mathrm{E}+\mathrm{Pi0}$ In A Large Water Cherenkov Detector, By the Super-Kamiokande Collaboration (M. Shiozawa et al.), Phys. Rev. Lett. 81, 3319-3323 (1998)

[15] Search For Nucleon Decay Using The IMB-3 Detector, By the IMB Collaboration (C. McGrew et al.), Phys. Rev. D59, 052004 (1999)

[16] Direct Evidence For Neutrino Flavor Transformation From Neutral Current Interactions In The Sudbury Neutrino Observatory, By the SNO Collaboration (Q.R. Ahmad et al.), Phys. Rev. Lett. 89, 011301 (2002)

[17] Determination Of Solar Neutrino Oscillation Parameters Using 1496 Days Of Super-Kamiokande-I Data, By the Super-Kamiokande Collaboration (S. Fukuda et al.), Phys. Lett. B539, 179-187 (2002)

[18] Solar Models: Current Epoch And Time Dependences, Neutrinos, And Helioseismological Properties, J.N. Bahcall, M.H. Pinsonneault, S. Basu, Astrophys. J. 555, 990-1012 (2001)

[19] Search For Supernova Relic Neutrinos At Super-Kamiokande, By the Super-Kamiokande Collaboration (M. Malek et al.), Phys. Rev. Lett. 90, 061101 (2003)

[20] Measuring The CP Violating Phase By A Long Baseline Neutrino Experiment With Hyper-Kamiokande, M. Aoki, K. Hagiwara, N. Okamura, Phys. Lett. B554 121-132 (2003)

[21] Supernova Pointing with Low- and High-energy Neutrino Detectors, R. Tomas, D. Semikoz, G.G. Raffelt, M. Kachelriess and A. S. Dighe, Phys. Rev. D 68, 093013 (2003)

[22] Detection Possibility of the Pair-annihilation Neutrinos from the Neutrino-cooled Pre-supernova Star, A. Odrzywolek, M. Misiaszek and M. Kutschera, Astropart. Phys. 21, 303 (2004)

[23] Detection Of The Free Neutrino: A Confirmation, C.L. Cowan, F. Reines, F.B. Harrison, H.W. Kruse, A.D. McGuire, Science 124, 103104 (1956)

[24] First Results From KamLAND: Evidence For Reactor Anti-neutrino Disappearance, By the KamLAND Collaboration (K. Eguchi et al.), Phys. Rev. Lett. 90, 021802 (2003) 
[25] Determination Of Neutrino Incoming Direction In The Chooz Experiment And Its Application To Supernova Explosion Location By Scintillator Detectors, M. Apollonio et al., Phys. Rev. D61, 012001 (2000)

[26] Reactor Anti-neutrino Oscillations And Gadolinium Loaded SuperKamiokande Detector, S. Choubey and S.T. Petcov, Phys. Lett. B594, 333-346 (2004) 